\title{
On the Smoluchowski paradox in a sedimenting suspension
}

\author{
C. W. J. Beenakker"a) and P. Mazur \\ Instituut-Lorentz, Rijksuniversiteit te Leiden, Nieuwsteeg 18, 2311 SB Leiden, The Netherlands
}

(Received 20 July 1984; accepted 26 September 1984)

It is shown, by explicit calculation, that the influence of a plane wall supporting the suspension on the sedimentation velocity is such that the convergence problems of this quantity encountered in an unbounded suspension do not occur-even in the limit of an infinitely distant wall.

\section{INTRODUCTION}

The motion of a particle in a viscous fluid causes a disturbance of the fluid flow which falls off very slowly with increasing distance to the particle, in fact only with the inverse first power of the distance. As a consequence, the influence of container walls on properties of suspensions can in certain cases not be neglected, even if the container is very large. An example, which forms a dramatic illustration, is the divergency of the velocity of sedimentation in an unbounded suspension. This paradoxical situation (which, first noticed in 1911 by Smoluchowski, ${ }^{1}$ has been referred to as the Smoluchowski paradox ${ }^{2}$ ) has received considerable attention. ${ }^{2-9}$ In 1972, Batchelor ${ }^{6}$ introduced an ingenious argument to resolve the difficulties, which has since become generally accepted-although not without controversy. ${ }^{10}$ The argument of Batchelor (to which we shall return) is based on general considerations of a physical nature and not on an explicit evaluation of the influence of container walls. As a matter of fact, such an explicit calculation woulduntil recently-not have been possible, because not enough was known about the interaction of particles via the fluid (the so-called hydrodynamic interaction) in the presence of a boundary wall.

It is the purpose of this note to present a explicit calculation of the sedimentation velocity for the most simple case: a dilute homogeneous layer of spherical particles sedimenting towards a plane wall, in an otherwise unbounded fluid. Our calculation is based on results from a study by van Saarloos and the authors ${ }^{11}$ of the hydrodynamic interactions between spheres and a wall. In the paper referred to, expressions for the mobility tensors of the spheres were obtained by an extension of a method developed previously for an unbounded fluid. ${ }^{12}$

The results for the mobilities, as far as necessary for our purpose, are given in Sec. II. In Sec. III we then calculate the (average) sedimentation velocity to linear order in the concentration of the suspended spheres, at a point sufficiently far from the wall supporting the fluid. A discussion follows in Sec. IV.

\footnotetext{
a) Present address: Department of Chemistry, Stanford University, Stanford, California 94305.
}

\section{RESULTS FROM THE HYDRODYNAMIC ANALYSIS}

We consider the motion of $N$ identical spherical particles with radius $a$ in an incompressible fluid with viscosity $\eta$, which is bounded by an infinite wall in the plane $z=0$. The centers of the spheres have positions $\mathbf{R}_{t}(i=1,2, \ldots N)$ and lie in the half-space $z>0$. We describe the motion of the fluid by the linear quasistatic Stokes equation, supplemented by stick boundary conditions on the surfaces of the spheres and on the wall.

The velocity $\mathbf{U}_{t}$ of sphere $i$ can be expressed as a linear combination of the forces $\mathbf{K}_{,}$, exerted by the fluid on each sphere $j$,

$$
\mathbf{U}_{i}=-\sum_{j=1}^{N} \boldsymbol{\mu}_{i j} \cdot \mathbf{K}_{j}, \quad i=1,2, \ldots N .
$$

(We have assumed here that the fluid exerts no torque on the spheres, i.e., each sphere can rotate freely.) General expressions for the mobility tensors $\mu_{i j}$ for this system have been obtained in Ref. 11, as an expansion in the two parameters $a /$ $R$ and $a /\left(R^{2}+4 l^{2}\right)^{1 / 2}$, where $R$ and $l$ are, respectively, the typical distances between two spheres and between a sphere and the wall. To third order one finds for the $z, z$ component of the mobility tensor (which is the quantity we shall use in the following):

$$
\begin{aligned}
& 6 \pi \eta a\left(\mu_{y}\right)_{z z}=\delta_{l y}\left[1-\frac{9}{8} a / l_{t}+\frac{1}{2}\left(a / l_{\imath}\right)^{3}\right] \\
& +\left(1-\delta_{\imath}\right)\left\{\frac{3}{4}\left(a / R_{\imath \jmath}\right)\left[1+\left(l_{J}-l_{t}\right)^{2} R_{\imath j}^{-2}\right]\right. \\
& +\frac{1}{2}\left(a / R_{\eta j}\right)^{3}\left[1-3\left(l_{J}-l_{l}\right)^{2} R_{\eta j}^{-2}\right] \\
& -\frac{3}{4}\left(a / \tilde{R}_{\imath \jmath}\right)\left[1+\left(l_{1}^{2}+l_{\jmath}^{2}\right) \tilde{R}_{\imath}^{-2}\right. \\
& \left.+6 l_{i} l_{j}\left(l_{\imath}+l_{j}\right)^{2} \tilde{R}_{\imath}^{-4}\right] \\
& -\frac{1}{2}\left(a / \tilde{R}_{\imath \jmath}\right)^{3}\left[1+6\left(l_{l}+l_{\jmath}\right)^{2} \tilde{R}_{\imath \jmath}^{-2}\right. \\
& \left.\left.-15\left(l_{t}+l_{\jmath}\right)^{4} \tilde{R}_{\imath j}^{-4}\right]\right\} \text {. }
\end{aligned}
$$

We have used here the notations $R_{i j} \equiv\left|\mathbf{R}_{j}-\mathbf{R}_{t}\right|$ and $\tilde{R}_{y} \equiv\left(R_{y}^{2}+4 l_{l} l_{j}\right)^{1 / 2}$, where $l_{i}$ and $l_{j}$ are the distances of the centers of, respectively, spheres $i$ and $j$ to the wall.

Note that to third order, $\mu_{u}$ depends on the positions of spheres $i$ and $j$ only. Higher-order terms, however, do contain contributions which depend on the positions of the other spheres as well, reflecting the nonadditivity of hydrodynam- 
ic interactions (cf. Refs. 11 and 12).

\section{CALCULATION OF THE SEDIMENTATION VELOCITY}

Consider a homogeneous layer (contained in the space $0<z<H$ ) of suspended spheres, sedimenting toward a horizontal plane wall at $z=0$ in an otherwise unbounded fluid. Because of translational invariance in the $x-y$ plane, the average velocity of sedimentation of the spheres $s$ is perpendicular to this plane and has a magnitude $s$ which depends on $z$ only, ${ }^{13}$

$$
\frac{s(z)}{s_{0}}=6 \pi \eta a \frac{1}{n(z)}\left\langle\sum_{i, j=1}^{N}\left(\mu_{i j}\right)_{z z} \delta\left(l_{i}-z\right)\right\rangle .
$$

Here $n(z) \equiv\left\langle\Sigma_{i} \delta\left(l_{i}-z\right)\right\rangle$ is the density profile, with the angular brackets denoting an average over the configurations of the spheres. The quantity $s_{0}$ is defined in terms of the gravitational force $F$ (corrected for bouyancy) on each of the spheres by $s_{0} \equiv(6 \pi \eta a)^{-1} F$, and is therefore the sedimentation velocity of an isolated sphere in an unbounded fluid.

Using the expression for the mobility given in Eq. (2), we shall calculate $s$ to linear order in the volume fraction $\phi$ of the suspended particles. (Note that to this order it is sufficient to consider only the contributions to the mobility which depend on the positions of at most two spheres.) Substituting Eq. (2) into Eq. (3) one finds

$$
\begin{aligned}
s(z) / s_{0}= & 1-\frac{9}{8} a / z+\frac{1}{2}(a / z)^{3} \\
& +\phi\left(\frac{4}{3} \pi a^{3}\right)^{-1} 2 \pi \int_{-\infty}^{\infty} d \zeta \int_{0}^{\infty} \rho d \rho \theta(R-2 a) \\
& \times \theta(\zeta+z-a) \\
& \times \theta(H-a-\zeta-z)\left\{\frac { 3 } { 4 } a \left[R^{-1}+\zeta^{2} R^{-3}-\tilde{R}-1\right.\right. \\
& -\left(\zeta^{2}+2 z \zeta+2 z^{2}\right) \tilde{R}-3 \\
& \left.-6 z(\zeta+z)(\zeta+2 z)^{2} \tilde{R}-5\right] \\
& +\frac{1}{2} a^{3}\left[R-3-3 \zeta^{2} R^{-5}-\tilde{R}-3\right. \\
& \left.\left.-6(\zeta+2 z)^{2} \tilde{R}-5+15(\zeta+2 z)^{4} \tilde{R}-7\right]\right\},
\end{aligned}
$$

where $\theta(x)$ is the step function (which equals one if $x>0$ and zero elsewise), and $R$ and $\tilde{R}$ are defined in terms of the polar coordinates $\rho$ and $\zeta$ by $R \equiv\left(\rho^{2}+\zeta^{2}\right)^{1 / 2}$, $\tilde{R} \equiv\left[\rho^{2}+(\xi+2 z)^{2}\right]^{1 / 2}$.

The radial integration in (4) over $\rho$ may be carried out straightforwardly and gives zero unless $|\xi|<2 a$. The final integration over $\zeta$ gives, for $3 a<z<H-3 a$,

$$
\begin{aligned}
s(z) / s_{0}= & 1-\frac{2}{8} a / z+\frac{1}{2}(a / z)^{3}+\mathscr{O}(a / z)^{4} \\
& +\phi\left[-5+9 a / z+\mathscr{O}(a / z)^{2}\right],
\end{aligned}
$$

independent of $H$. If we now take the limit $z \rightarrow \infty$ of Eq. (5) (after having taken the limit $H \rightarrow \infty$ ), we recover the result of Batchelor ${ }^{6}$ for the influence of long-ranged hydrodynamic interactions on the sedimentation velocity. If, however, one would have taken these limits before the integration in $\mathrm{Eq}$. (4), one would have found a divergent sedimentation velocity upon integration. This is, in mathematical terms, the Smoluchowski paradox mentioned in the introduction.

Three further remarks are in order: firstly, we note that Batchelor ${ }^{6}$ has also calculated that short-ranged hydrody- namic interactions between two spheres [which were not included in the expression to third order (2), but could easily have been taken into account as well, cf., e.g., Ref. 11] give an additional contribution of $-1.55 \phi$ to the rhs of Eq. (5). Secondly, we observe that the correction of order $a / z$ to the sedimentation velocity from the wall supporting the fluid has a coefficient $-9 / 8+9 \phi+\mathscr{O}\left(\phi^{2}\right)$; whereas in this order the wall retards sedimentation of an isolated sphere (which is the well-known result of Lorent ${ }^{14}$ ), it has an enhancing effect via the hydrodynamic interactions between two spheres. Thirdly, one may also evaluate (using the expression for the mobility given in Ref. 11) the velocity of sedimentation parallel to the wall, and show that this velocity then diverges in the limit $H \rightarrow \infty$, as it does in the case of an unbounded fluid. This is to be expected, since no adequate backflow can be generated in this case.

\section{DISCUSSION}

In the previous section we have shown, to the order considered, that the influence of the wall supporting the suspension on the mobility tensors is such that the convergence problems of the sedimentation velocity encountered in an unbounded fluid do not occur-even in the limit of an infinitely distant wall.

In an unbounded medium, the difficulties encountered are of two kinds. First, there is a divergent contribution due to the $R^{-1}$ term in the hydrodynamic interaction of two spheres, a distance $R$ apart. Second, there is a conditionally convergent (and, therefore, shape dependent) contribution due to the $R^{-3}$ interaction. The first difficulty was resolved by Pyun and Fixman ${ }^{5}$ by considering sedimentation with respect to the mean volume-velocity. Batchelor ${ }^{6}$ was able to assign a definite value to the resulting shape-dependent expression (thereby resolving the second difficulty) by requiring that $(\partial / \partial \mathbf{r}) \cdot \boldsymbol{\sigma}(\mathbf{r})$ be zero on average, where $\boldsymbol{\sigma}(\mathbf{r})$ (defined for all $\mathbf{r}$ ) is the traceless part of the pressure tensor. This physical requirement prescribes the integration volume for the conditionally convergent integral, which turns out to be the volume enclosed between two horizontal planes. As we have seen, the result of Batchelor (rederived in Refs. 7 and 8 by alternative arguments of the same nature) agrees with that from the explicit calculation presented here.

It would be interesting to calculate the influence of container walls on the sedimentation velocity also for a different geometry than that considered here (for example, for a vertical cylinder which is closed at the bottom end), in order to verify that sedimentation far from boundaries does not depend on the shape of the container, as argued by Batchelor and others. ${ }^{6-8}$

Within the general context of this problem, we mention the work of Hurd ${ }^{2}$ and Goren ${ }^{9}$ on the motion of a linear array of spheres, lying with equal separations $R$ on a line parallel to a plane wall (at a distance $l$ from the wall). Whereas Hurd considers only motion along the line of centers, Goren studies motion in arbitrary directions. For small values of the ratio $l / R$ (corresponding to the regime of validity of the theory ${ }^{9}$ ), finite answers are found for the mobility of the array, even in the case of motion parallel to the wall. This last result is in marked contrast to what is found for a three- 
dimensional suspension, cf. the last remark of Sec. III. Thus, while these theories ${ }^{2,9}$ are highly interesting as such, it must be doubted whether they have a direct bearing on the problem of a sedimenting suspension studied in this paper.

As a final remark, we draw attention to the fact that in a possible extension of our calculation to terms of second power in the concentration, it would be essential to account for the nonadditivity of hydrodynamic interactions. Indeed, two- and three-sphere interactions have been shown (in the context of diffusion) to give contributions of second power in the concentration of comparable magnitude. ${ }^{15}$

\section{ACKNOWLEDGMENTS}

We thank U. Geigenmüller for fruitful discussions on the subject of this paper and one of the referees for drawing our attention to Ref. 9.

This work was performed as part of the research program of the "Stichting voor Fundamenteel Onderzoek der Materie" (F.O.M.) with financial support from the "Nederlandse Organisatie voor Zuiver-Wetenschappelijk Onderzoek" (Z.W.O.).
'M. Smoluchowski, Bull. Acad. Sci. Cracow 1a, 28 (1911).

${ }^{2}$ A. J. Hurd, Ph.D. thesis, University of Colorado, Boulder, 1981.

${ }^{3}$ M. Smoluchowski, Proc. 5th Intern. Congr. Math. Cambridge 2, 192 (1912).

${ }^{4} J$. M. Burgers, Proc. Kon. Nederl. Akad. Wet. 44, 1045, 1177 (1941); 45, 9, $126(1942)$.

${ }^{5} \mathrm{C}$. W. Pyun and M. Fixman, J. Chem. Phys. 41, 937 (1964).

${ }^{6}$ G. K. Batchelor, J. Fluid Mech. 52, 245 (1972).

${ }^{7}$ E. J. Hinch, J. Fluid Mech. 83, 695 (1977).

${ }^{8}$ A. B. Glendinning and W. B. Russel, J. Colloid Interface Sci. 89, 124 (1982).

${ }^{9}$ S. L. Goren, J. Fluid Mech. 132, 185 (1983).

${ }^{10}$ R. Herczyński and I. Pieńkowska, Ann. Rev. Fluid Mech. 12, 237 (1980).

${ }^{11} \mathrm{C}$. W. J. Beenakker, W. van Saarloos, and P. Mazur, Physica A 127, 451 (1984).

${ }^{12}$ P. Mazur and W. van Saarloos, Physica A 115, 21 (1982).

${ }^{13}$ The influence of Brownian motion on the sedimentation velocity is ignored in this paper. In fact one can show that this influence vanishes in the linear density regime considered; see G. K. Batchelor, J. Fluid Mech. 119, 379 (1982).

${ }^{14}$ H. A. Lorentz, Abhandlungen über theoretische Physik I (Teubner, Leipzig, 1907).

${ }^{15}$ C. W. J. Beenakker and P. Mazur, Phys. Lett. 91A, 290 (1982), Physica A 120, 388 (1983); A 126, 349 (1984). 